\title{
Spezialisierung und Fragmentierung sind zweierlei
}

\section{Werner Bauer}

Dr. med., Präsident des Schweizerischen Instituts für ärztliche Weiter- und Fortbildung SIWF

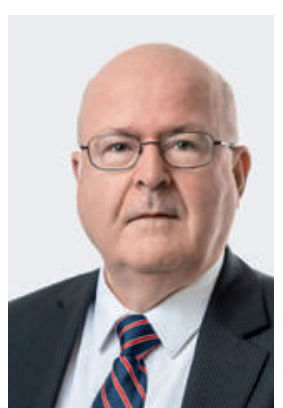

«Nimmt die unselige Spezialisierung denn nie ein Ende?», «Schluss jetzt mit der Schaffung neuer Titel!»solche Stossseufzer hört man immer wieder. Sie zeugen vom Unbehagen über die zunehmende Zersplitterung von Fachgebieten, weil neue Methoden anerkannt werden wollen oder weil Teilbereiche eigene Berufsbilder und eigene Kompetenzen entwickeln.

Die berufliche Spezialisierung ist allerdings kein neues Phänomen. Sie hat schon begonnen, als der Mensch anfing, Techniken zu suchen, um die anfallenden Arbeiten effizienter zu bewältigen - im Handwerk, in der Landwirtschaft, im Militärwesen und früh schon auch in der Medizin:

\section{Eine Spezialisierung in der Medizin ist} notwendig und unvermeidbar ...

In seinem Buch Das Leben im alten Rom (2. Auflage 1961, Francke) schreibt Ugo Enrico Paoli: «Es gab Spezialisten und Ärzte für allgemeine Krankheiten, jedoch überwogen die ersten [!]. Die ärztliche Zuständigkeit war weitgehend aufgeteilt und ging eigentlich weit über die Grenzen des Notwendigen und Vernünftigen hinaus. Es gab nicht nur Spezialisten für Ohren-, Augen-, Zahn-, Hals- und Frauenkrankheiten, sondern auch für Fieber, Schwindsucht usw. und unter den Chirurgen für Amputationen, Verwundungen, Brüche und Massage. Von dem berühmten Chirurgen Alco, der zur flavischen Zeit lebte, weiss man, dass er hauptsächlich Leistenbrüche und Knochenerkrankungen behandelte.»

Sir William Osler stellte am Ende des 19. Jahrhunderts schon fest, was auch heute noch zutrifft: «It is almost unnecessary to remark that the public, in which we live and move, has not been slow to recognize the advantage of a divison of labor in the field of medicine.»

Osler macht aber einen klaren Vorbehalt gegenüber zu weit gehender Spezialisierung: «Specialism is not, however, without many disadvantages. A radical error at the onset is the failure to recognize that the results of specialized observation are at best only partial truths, which require to be correlated with facts obtained by wider study. The various organs are not isolated, but complex parts of a complex whole, and every day's experience brings home the truth of the saying: When one member suffers all the members suffer with it" (Remarks on Specialism. Boston Med Surg J. 1892;126:457-9).

Auch diese historischen Zitate bestätigen, dass die Spezialisierung seit je zwei Seiten hat: Zum einen sind Spezialisten beim Publikum durchaus gesucht, und neue, anspruchsvolle Methoden sowie spezialisierte Arbeitsbereiche müssen hinsichtlich Weiterbildung und einwandfreier Berufsausübung öffentlich erkennbar definiert werden. Der medizinische Fortschritt wird unweigerlich weitere neue Methoden und auch die Notwendigkeit bringen, Berufsbilder zu revidieren oder neu zu schaffen. Aktuelle Beispiele sind die soeben genehmigten Schwerpunkte Palliativmedizin sowie Psychiatrie und Psychotherapie der Abhängigkeitserkrankungen. Das Schweizerische Institut für ärztliche Weiter- und Fortbildung schafft neue Titel keinesfalls unbesehen und klärt nicht nur die fachliche Begründung der Anträge $a b$, sondern achtet darauf, ob eine neue Qualifikation für die Patientenversorgung sinnvoll und mit den Strukturen des Gesundheitswesens kompatibel ist. Dazu gehört immer

... sie muss aber auch den zentralen Stellenwert der Generalisten berücksichtigen.

auch der Blick nach Europa. Zum anderen sind die Schattenseiten einer zu weit gehenden Spezialisierung ebenfalls bekannt: Sie kann zu einer zunehmenden Fragmentierung von Abläufen und Strukturen führen. Sie droht auch, die Generalisten zu marginalisieren, die es in vielen Fällen zwingend braucht, um Patienten integrierend und priorisierend zu betreuen.

Auf Anregung aus Spitalkreisen wird innerhalb der BAG-GDK-Plattform "Ärztliche Bildung» eine Arbeitsgruppe «Zunehmende Spezialisierung in der Humanmedizin aus Sicht der Spitalorganisation" gebildet, um die aktuelle Situation, die Perspektiven und deren Konsequenzen $\mathrm{zu}$ analysieren. Um eine Feststellung wird auch sie nicht herumkommen: Jedes Ding hat zwei Seiten. 